\title{
Characteristics of Real Futures Trading Networks
}

\author{
Junjie Wang ${ }^{\mathrm{a}, \mathrm{b}}$, Shuigeng Zhou ${ }^{\mathrm{a}, \mathrm{c}, *}$, Jihong Guan ${ }^{\mathrm{d}, *}$ \\ ${ }^{a}$ School of Computer Science, Fudan University, Shanghai 200433, China \\ ${ }^{b}$ Shanghai Futures Exchange, Shanghai 200122, China \\ ${ }^{c}$ Shanghai Key Lab of Intelligent Information Processing, Fudan University, Shanghai 200433, China \\ ${ }^{d}$ Department of Computer Science and Technology, Tongji University, Shanghai 201804, China
}

\begin{abstract}
Futures trading is the core of futures business, and it is considered as one of the typical complex systems. To investigate the complexity of futures trading, we employ the analytical method of complex networks. First, we use real trading records from the Shanghai Futures Exchange to construct futures trading networks, in which nodes are trading participants, and two nodes have a common edge if the two corresponding investors appear simultaneously in at least one trading record as a purchaser and a seller respectively. Then, we conduct a comprehensive statistical analysis on the constructed futures trading networks. Empirical results show that the futures trading networks exhibit features such as scale-free behavior with interesting odd-even-degree divergence in low-degree regions, small-world effect, hierarchical organization, power-law betweenness distribution, disassortative mixing, and shrinkage of both the average path length and the diameter as network size increases. To the best of our knowledge, this is the first work that uses real data to study futures trading networks, and we argue that the research results can shed light on the nature of real futures business.
\end{abstract}

Keywords: Complex networks, Futures trading networks, Scale-free scaling, Small-world effect PACS: 89.75.Fb, 89.75.Hc, 89.65.Gh

\section{Introduction}

Since the works of Watts \& Strogatz [1] and Barabási \& Alberta [2] were published, complex networks, as a new scientific area, have attracted a tremendous amount of research interest [37]. Complex networks can describe a wide range of real-life systems in nature and society, and show various nontrivial topological characteristics not occurring in simple networks such as regular lattices and random networks. There are a number of frequently cited examples that have been studied from the perspective of complex networks, including the World Wide Web [811], the Internet [12], metabolic networks [13], scientific collaboration networks [14, 15], online social networks [16, 17], public transport networks [18, 19], airline flight networks [15, 20-23] and human language networks [24-26]. Empirical studies on these networks mentioned above

\footnotetext{
${ }^{*}$ Corresponding author

Email addresses: wangjunjie@fudan.edu.cn (Junjie Wang), sgzhou@fudan.edu.cn (Shuigeng Zhou), jhguan@tongji.edu.cn (Jihong Guan)
}

Preprint submitted to Physics A 
have largely motivated the recent curiosity and concern about this new focus of research so that a number of techniques and models have been explored to improve people's perception of topology and evolution of real complex systems [27-34]. With growing of importance and popularity, complex network theory has become a powerful tool with intuitive and effective representations to analyze complex systems in a variety of fields, including financial markets [35].

In the literature, a number of papers have been dedicated to studying financial markets from the perspective of complex networks. The major difference among these works lies in the types of networks to be constructed from financial data for characterizing the organization and structure of financial markets. Some existing works constructed stock networks whose connectivity is defined by the correlation between any two time series of stock prices [36-40]. Some others established directed networks of stock ownership describing the relationship between stockholders and companies [41, 42]. Networks of market investors based on transaction interactions between the investors were also investigated. For example, Franke et al. [43] analyzed irregular trading behaviors of users in an experimental stock market, while Wang et al. [44] studied the evolving topology of such a network in an experimental futures exchange. Recently, Jiang et al. [45] has investigated stock weighted directed trading networks based on daily transaction records through reconstructing the limit order book with real order series from the Shenzhen Stock Exchange.

The study on financial investor networks can provide clues for revealing the true complexity in financial markets, especially futures markets. In a real futures market, the futures trading model serves as a matching engine for executing all eligible orders from various market participants, and the interactions among the participants form a complex exchange network, which is termed as the futures trading network (FTN in short) in this paper. Simply put, a FTN consists of a set of trading participants, each of which has at least one connection of direct exchange action to another by futures contract(s).

In this paper, we try to provide a comprehensive study on the characteristics of futures trading networks established with genuine trading data from the Shanghai Futures Exchange. To the best of our knowledge, this is the first work that uses real futures trading data for constructing networks. So we assume that the empirical results may unveil to some degree futures trading behaviors and shed light on the nature of futures markets.

The rest of this paper is organized as follows. The FTN construction method is introduced in Section 2, including trading dataset and the details of network construction. Section 3 presents the empirical results of FTNs. Section 4 concludes the paper and highlights some future work directions.

\section{Construction of Futures Trading Networks}

In this section, we first introduce the real trading data from the Shanghai Futures Exchange, which will be used for constructing futures trading networks, and then present the details of the construction of futures trading networks.

\subsection{Dataset}

For constructing the futures trading networks, we use real trading records from the Shanghai Futures Exchange, which is the largest one in China's domestic futures market and has considerable impact on the global derivative market. Trade records are generated by matching orders or quotes from buyers and sellers according to a certain rule of price/time priority (first price, then time) via the electronic trading platform of the futures exchange. There are hundreds of 
thousands of matching results reported from the exchange in one typical trading day. We use a dataset involving all futures commodities in the derivative market from July to September, 2008. For the trading records, we use virtual and unique IDs for representing the trading participants, and all other sensitive information is filtered for privacy preservation reason.

\subsection{Network Construction}

Since a trading record contains the IDs of both the buyer and the seller, we are able to establish a futures trading network. The construction process is as follows. A node represents a participant in a trading record, and an edge, meaning a trade relation, is established between two participants if their IDs appear simultaneously at least once in one trading record. An example of FTN that comprises nine records is shown in Fig. 1. Here, A-H are IDs of trading participants. Each row in the right table of Fig. 1 represents a trading record where the seller and buyer columns are the IDs of seller and buyer.

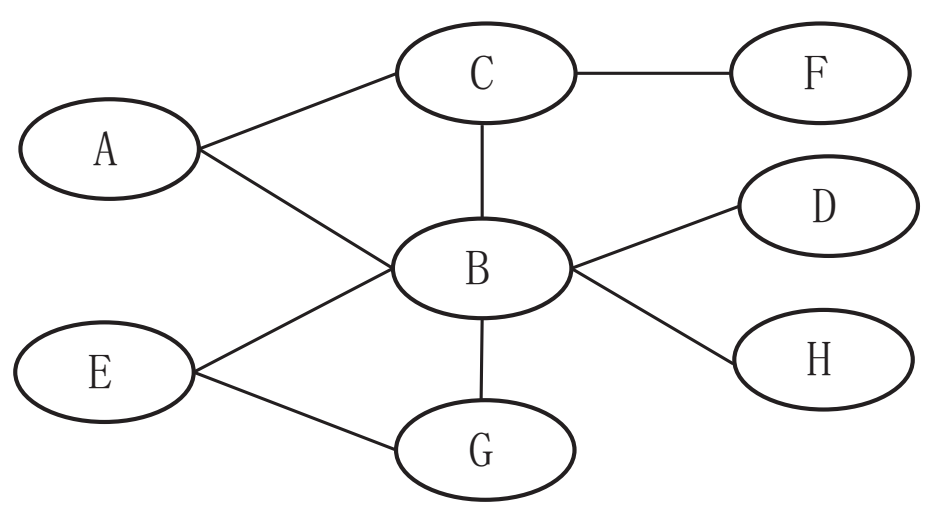

\begin{tabular}{cc}
\hline seller & buyer \\
\hline A & B \\
A & C \\
B & C \\
B & D \\
B & E \\
B & G \\
B & H \\
C & F \\
E & G \\
\hline
\end{tabular}

Fig. 1. An example of the futures trading network (left) constructed from a dataset with nine trading records (right).

Following the above process, we first create the largest network with the whole dataset containing three months' trading records; this network is denoted as FTN-all. Then, we construct three sub-networks based on three subsets of the whole dataset above according to the futures commodity classification of the Shanghai Futures Exchange. These three sub-networks are termed as FTN-met, FTN-rub and FTN-oil, which involve only futures commodity metal (concretely including copper, aluminum, zinc and gold), natural rubber and fuel oil, respectively. Thus, we have totally four FTNs, their statistic information is given in Table 1.

\section{Properties of Futures Trading Networks}

In what follows, we study the characteristics of FTNs constructed above, our focus is on topological features and dynamical properties. Extensive empirical results are presented. 


\subsection{Scale-free Behavior}

The degree distribution $P(k)$ is one of the most important statistical characteristics of a network, and also one of the simplest properties that can be measured directly. It is defined as the probability that a random node in a network has exactly $k$ edges. In many real complex networks, $P(k)$ decays with $k$ in a power law, following

$$
P(k) \sim k^{-\lambda} .
$$

A network that owns such a property is called a scale-free network [2]. We plot the degree distributions $P(k)$ of FTN-all, FTN-met, FTN-rub and FTN-oil in Fig. 2(a)-(d). Our observation of the subplots implies that all the four FTNs are scale-free networks. However, reaching a conclusion that all FTNs are scale-free requires a deeper analysis by statistic techniques.

To detect the existence of power-law property in FTNs, we consider the cumulative degree distribution function defined as $P_{\text {cum }}(k)=\operatorname{Pr}\left(k^{\prime} \geq k\right)$ because the power-law cumulative degree distribution of a network means that its degree distribution obeys a power law [46]. According to Ref. [47], we calculate the complementary cumulative degree distributions of FTNs for estimating the scaling parameter $\lambda$ and the lower bound $k_{\min }$ of power-law behavior based on the methods of maximum likelihood estimators and the quantification of the distance between two probability distributions. Furthermore, we conduct a goodness-of-fit test by computing the distance between the empirical distribution of FTN and the power-law model with the Kolmogorov-Smirnov (KS) statistic, and generating a $p$-value that quantifies the plausibility of a power-law degree distribution hypothesis for each FTN. The cumulative degree distributions $P_{\text {cum }}(k)$ for all the four networks are presented in Fig. 3 with the slope $(\lambda-1)$. Table 2 gives the estimations of $\lambda, k_{\min }$, and the $p$-value of the goodness-of-fit test for each power-law fit of FTN.

As listed in Table 2, the scaling parameters $\lambda$ of the four FTNs have nearly similar values of 2.48, 2.39, 2.53 and 2.39 for FTN-all, FTN-met, FTN-run and FTN-oil, respectively. Their

Table 1. The statistic information of the four FTNs. FTN-all is based on the whole dataset containing three months' trading records from July to September, 2008, FTN-met, FTN-rub and FTN-oil are three sub-networks of FTN-all that correspond to three subsets that involve only trading records of the futures commodity metal, natural rubber and fuel oil, respectively.

\begin{tabular}{lccc}
\hline Network & Futures commodity & Number of nodes & Number of edges \\
\hline FTN-all & all commodities & 100994 & 8068676 \\
FTN-met & metal & 75262 & 3226119 \\
FTN-rub & natural rubber & 55828 & 3364557 \\
FTN-oil & fuel oil & 52208 & 1657268 \\
\hline
\end{tabular}

Table 2. The estimations of the lower bound $k_{\min }$ and the scaling parameter $\lambda$, and the goodness-of-fit tests of power-law behavior for the four futures trading networks. The $p$-values indicate that the degree distribution of each network is consistent with a power-law hypothesis.

\begin{tabular}{lccc}
\hline Network & $k_{\min }$ & $\lambda$ & $p$-value \\
\hline FTN-all & 926 & 2.48 & 0.225 \\
FTN-met & 339 & 2.39 & 0.106 \\
FTN-rub & 1196 & 2.53 & 0.591 \\
FTN-oil & 351 & 2.39 & 0.674 \\
\hline
\end{tabular}



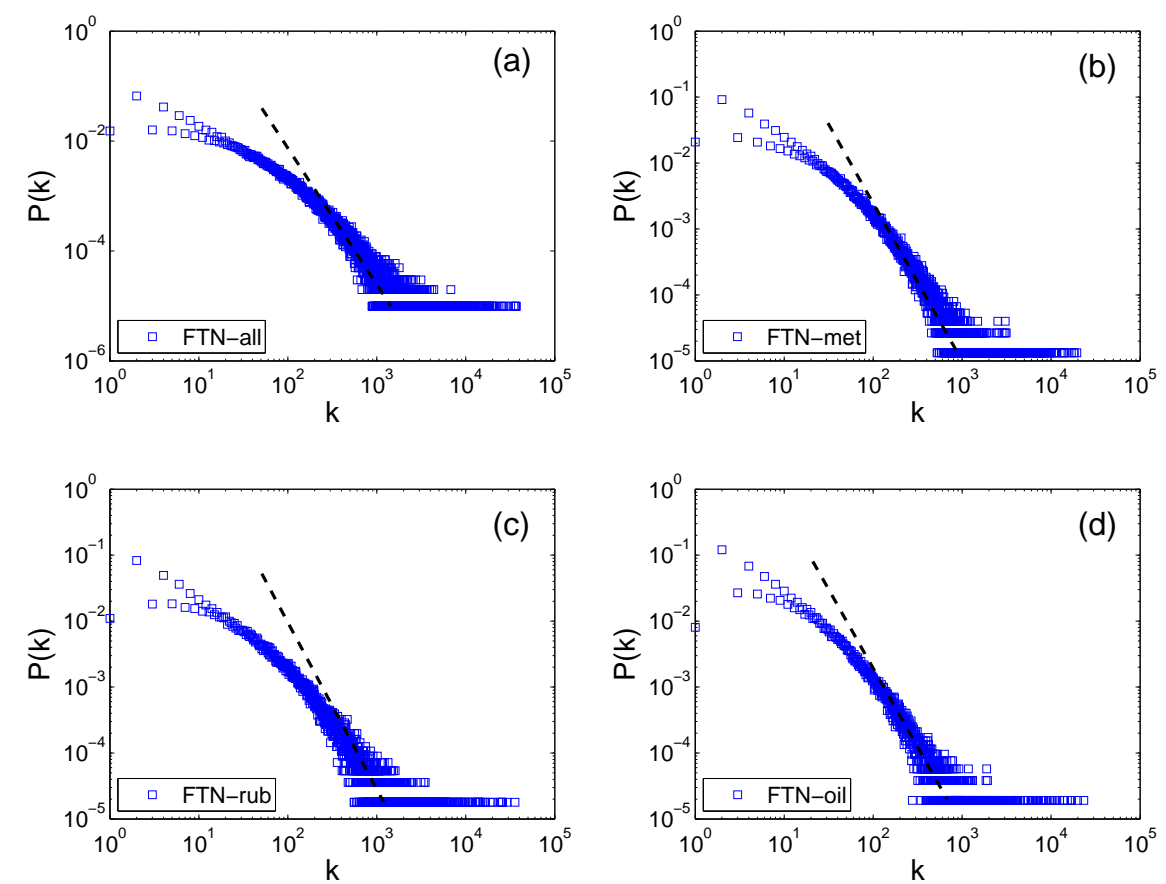

Fig. 2. The degree distributions $P(k)$ of the four futures trading networks FTN-all, FTN-met, FTN-rub and FTN-oil. All the four fitting lines of the subplots have nearly identical slopes, and the scaling parameters $\lambda$ are $2.48,2.39,2.53$ and 2.39 , respectively.

$p$-values are $0.225,0.106,0.591$ and 0.674 , respectively, which provide sufficient evidence indicating that the power law is a plausible hypothesis for the degree distribution of each FTN. These results imply that highly connected nodes have larger possibilities of happening and dominating the connectivity of FTNs. Actually, these nodes correspond to a few active speculators who issue numerous orders to the exchange, and consequently have more opportunities to make deals with the others. Their trading behaviors also account for the fact that FTNs dynamically expand in accordance with the rule of preferential attachment by continuously adding new nodes during the lifetime of a network.

In Fig. 2, we can also see the fact that in low-degree regions, the probability of even degree (say $k=2 n$ ) is larger than that of odd degree $(k=2 n+1)$, which forms a divergence. As the degree increases, the two branches gradually reach to a convergence and the difference is averaged out. The branch of even degree conforms to power law more than that of the odd one. One reasonable explanation on this observation is that most participants of low degree, entering the futures market as speculators, cautiously make a few transactions; but worrying about risk or loss, they do not hold the positions long, that is, they open futures positions(buying/selling) and close them soon (selling/buying). Certainly, there are some newcomers who are market investors and prefer holding positions for long-term profits. To a certain extent, due to the randomicity of matching counterpart during trade execution, the two matches (open and close) for a speculator in 

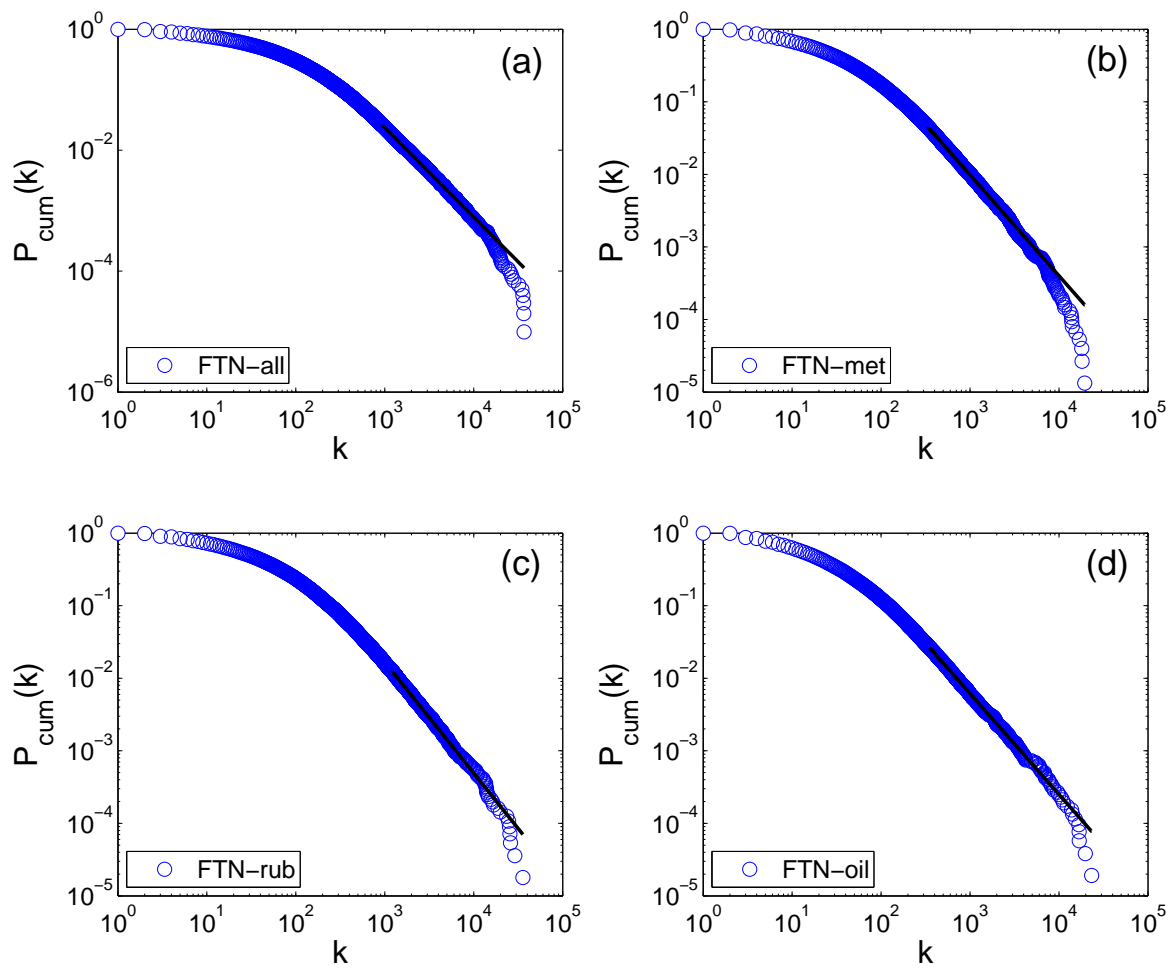

Fig. 3. The cumulative degree distributions $P_{\text {cum }}(k)$ and their maximum likelihood power-law fits for FTNall, FTN-met, FTN-rub and FTN-oil. The slopes of all fitting lines of the subplots are approximately similar, with values of $1.48,1.39,1.53$ and 1.39 , respectively.

a complete open and close are very possibly involved in different counterparts; thus two degrees (edges) will be added to the corresponding node. This observation indicates that there are few hedgers with long-term positions in real trading, which is consistent with the fact of low delivery ratio in the futures market.

Now we consider weighted futures trading networks in which our research focus is the relationship between strength and degree of nodes. The strength-degree relation in air transportation networks [15, 20-23] represents a power law, and various models [48-52] were proposed to explain the origin of the power-law correlation. In this paper, the node strength is defined as follows. Suppose that the weight of an edge between two nodes is the number of times they appear in the same trade record, the strength of a node is defined as the total weight of all edges connecting it. Fig. 4 shows the relationship between the average strength $S$ of the nodes with degree $k$ and $k$ in the four FTNs, which indicates that a nontrivial power-law scaling $S \sim k^{\beta}(\beta=1.12$ for all the four networks) exists, and demonstrates the fact that active market participants get more active. Such a scale-free behavior being correlated to the occurrence frequency of a trading participant provides another justification for the power-law degree distribution of the networks. 

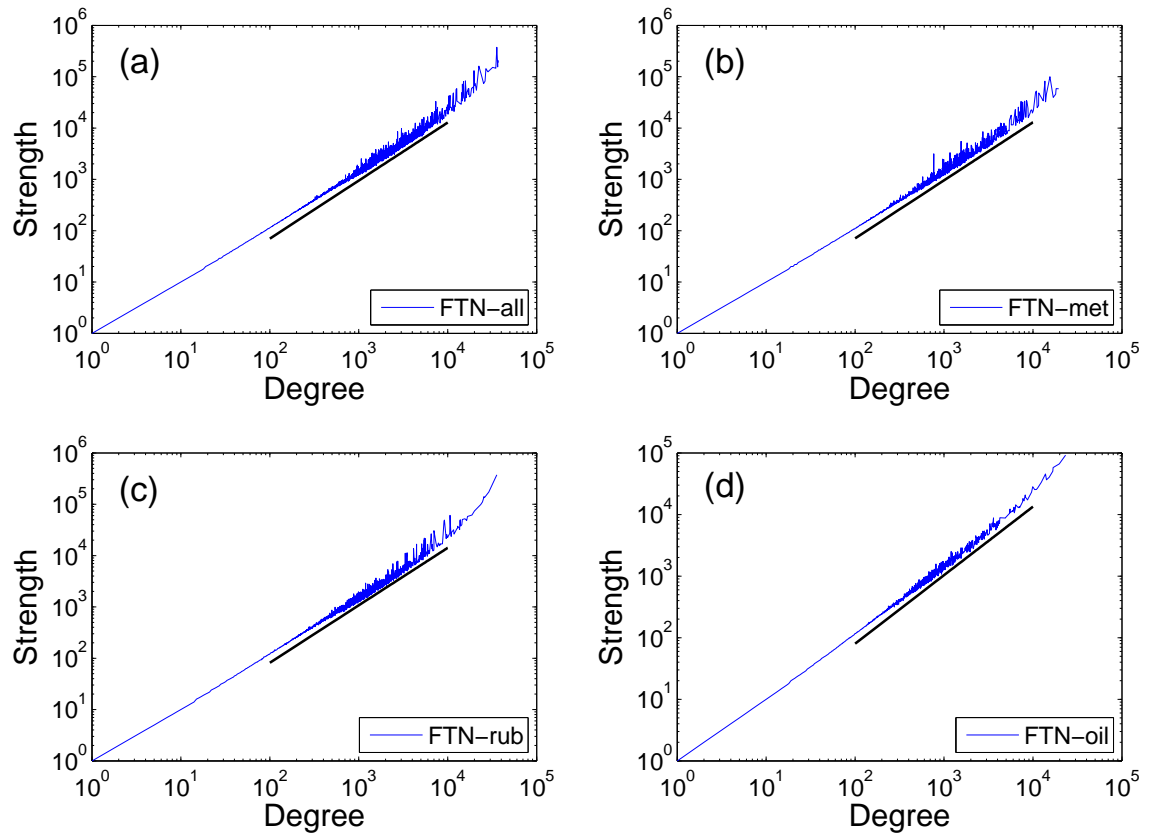

Fig. 4. Node strength $S$ versus degree $k$ for FTN-all, FTN-met, FTN-rub and FTN-oil. Here, the slopes are nearly similar to $1.12 \pm 0.01$. 


\subsection{Small-world Effect}

For complex networks, the average path length and the clustering coefficient are two important measures of small-world effect.

In a network, the average path length (say $L$ ) is defined as the number of edges in the shortest path between any two nodes, averaged over all pairs of nodes. It plays an important role in transportation and communication of a network. We can obtain $L=(2 / n(n-1)) \sum_{i \geq j} d_{i j}$. The longest shortest path among all pairs of nodes is called the diameter of the network.

The clustering coefficient $C_{i}$ of a node $i$ is defined as the ratio of the total number $e_{i}$ of edges that actually exist between all its $k_{i}$ immediate (nearest) neighbors over the number $k_{i}\left(k_{i}-1\right) / 2$ of all possible edges between them, that is, $C_{i}=2 e_{i} / k_{i}\left(k_{i}-1\right)$. The clustering coefficient $C$ of the whole network is the average of $C_{i}$ over all nodes, i.e., $C=(1 / n) \sum C_{i}$. The clustering coefficient measures the probability that two neighbors of a node are connected and reveals the local cliquishness of a typical neighborhood within a network.

In recent empirical studies, many real systems show the small-world effect by two crucial factors: the average path length and the diameter is relatively small despite often the large network size, which grows nearly logarithmically with the number of nodes, and the clustering coefficient is larger than that of a reference random network having the same number of nodes and edges as the real network.

We also notice the small-world effect in the four FTNs. On one hand, the average path length $L$ and the diameter $D$ are small. For the FTNs, $L$ ranges from 2.307 to 2.642 , while the values are between 2.774 and 2.933 in the corresponding equivalent random networks generated by the Erdos-Renyi model with the same parameters from each FTN. The diameters $D$ in the FTNs are 5 or 6 , while 3 or 4 in the corresponding random networks. On the other hand, these networks are highly clustered. The clustering coefficients $C$ are $0.0480,0.0453,0.0499$ and 0.0370 for FTN-all, FTN-met, FTN-rub and FTN-oil, respectively, and the value of each FTN is larger than that of the corresponding random network (with values of 0.0016, 0.0011, 0.0022 and 0.0012 , respectively). These results are presented in Table 3.

Table 3. Small-world effect is seen in all the four futures trading networks with relatively large clustering coefficient $C$ and small average path length $L$, contrary to the corresponding random networks generated by the Erdos-Renyi model with the same parameters (total number of nodes $N$, total number of edges $E$ and average number of edges per node $\langle k>$ ) of the futures trading networks.

\begin{tabular}{lccccccc}
\hline Network & $N$ & $E$ & $\langle k\rangle$ & $k_{\max }$ & $C$ & $L$ & $D$ \\
\hline FTN-all & 100994 & 8068676 & 159.8 & 36878 & 0.0480 & 2.470 & 6 \\
random & - & - & - & 218 & 0.0016 & 2.774 & 4 \\
FTN-met & 75262 & 3226119 & 85.7 & 19466 & 0.0453 & 2.642 & 6 \\
random & - & - & - & 133 & 0.0011 & 2.905 & 4 \\
FTN-rub & 55828 & 3364557 & 120.5 & 35694 & 0.0499 & 2.307 & 6 \\
random & - & - & - & 169 & 0.0022 & 2.766 & 3 \\
FTN-oil & 52208 & 1657268 & 63.5 & 23286 & 0.0370 & 2.494 & 5 \\
random & - & - & - & 102 & 0.0012 & 2.933 & 4 \\
\hline
\end{tabular}

The ratio of each FTN's clustering coefficient over that of its corresponding random network is relatively small in comparison with other real systems [4]. This implies no adequate evidence of local cliquishness of a typical neighborhood within each FTN, which may be due to the randomicity of matching counterpart during trade execution. Furthermore, the small $L$ and 
$D$ is because of 1) the existence of hub nodes (conforming to the large maximum degree $k_{\text {max }}$ in Table 3) that are bridges between different nodes in the networks, and 2) new connections generated between the existing nodes previously without direct links, which provide more shortcuts to the networks. The shortest path distribution in Fig. 5 shows that most of the shortest paths in the four networks are 2 or 3 in length, which helps explaining the small-world effect.
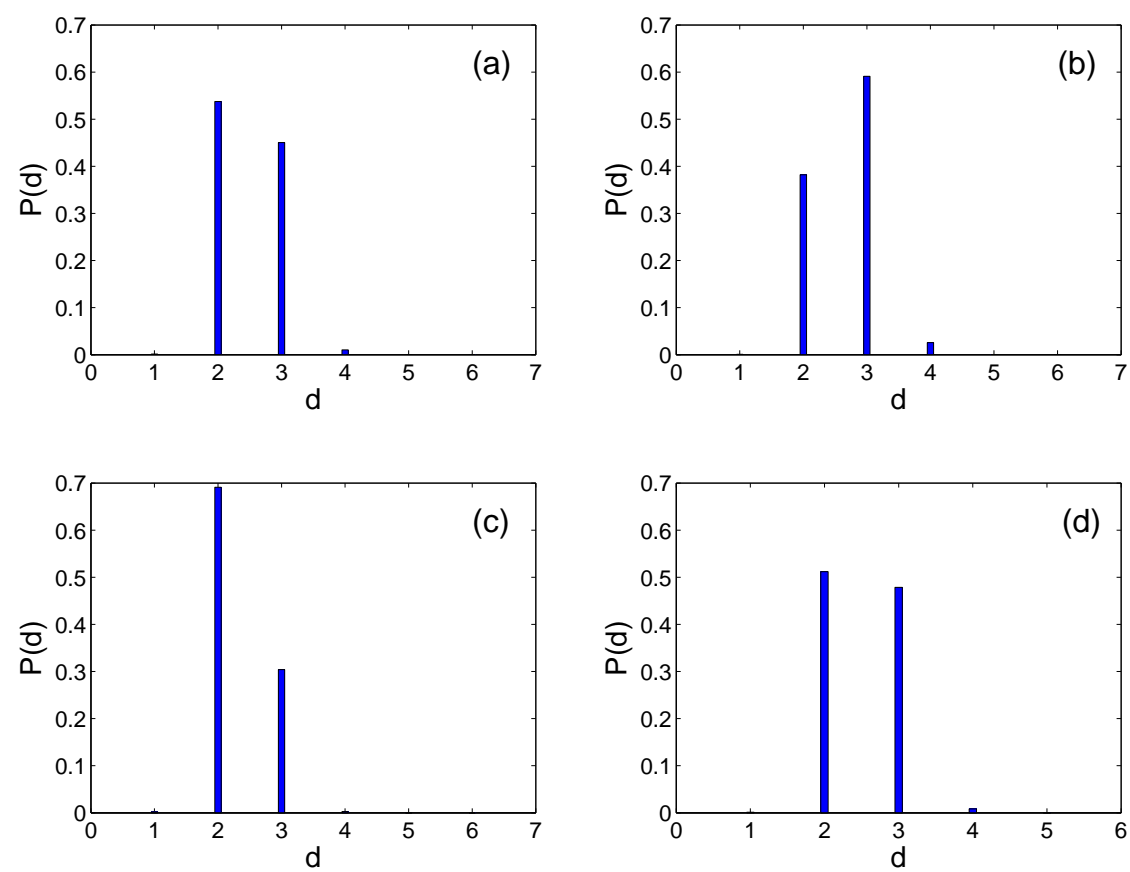

Fig. 5. The shortest path distributions $P(b)$ of the four futures trading networks. Obviously, most of the shortest paths of each network are 2 or 3 in length.

\subsection{Hierarchical Organization}

To examine the hierarchical organization feature of a FTN, we check $C(k)$, the average clustering coefficient of all nodes with the same degree $k$. If $C(k)$ follows a strict scaling law, then hierarchical organization exists in the network. Some real networks, such as the World Wide Web, the actor network and the Internet, display hierarchical topologies [53].

For each FTN, as indicated in Fig. 6, the main part of $C(k)$ obeys a scaling law of

$$
C(k) \sim(k+b)^{-\beta}
$$

with nearly similar exponent $\beta=0.87 \pm 0.03$, which implies that the network has a hierarchical architecture. Although the majority of bargainers who make a few deals have a few links (low $k$ ), most of these links join hub nodes that connect to each other, resulting in a big $C(k)$. The high- $k$ nodes are hub bargainers, their neighbors that are low- $k$ nodes are seldom linked to each 
other, leading to a smaller $C(k)$. This implies that ordinary investors are part of such clusters with high cohesiveness and dense interlinking, and the hubs play a bridging role to connect many separate small communities together into a complete network. To some extent, the hub speculators' trading behaviors flourish the futures market by improving market liquidity, and consequently enhance the pricing function of the futures market.
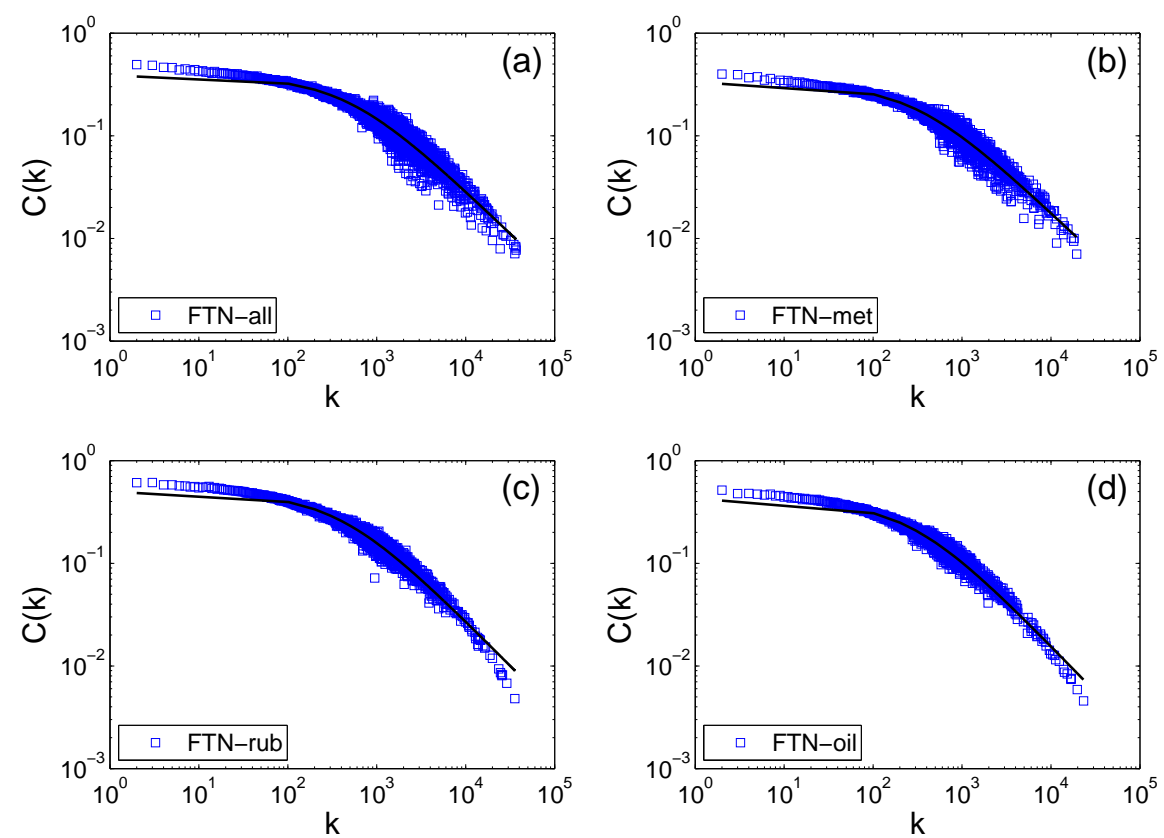

Fig. 6. Clustering coefficient $C(k)$ as a function $C(k) \sim(k+b)^{-\beta}$ of node degree $k$ with nearly similar exponent of $0.87 \pm 0.03$ for the four futures trading networks, which implies that each network has a hierarchical architecture.

\subsection{Betweenness Distribution}

Betweenness is a measure of the centrality of a node in a network; it is also a measure of influence that a node has over the spread of information in the network [54].

The betweenness of a given node is defined as the sum of the fraction of shortest paths between all pairs of nodes in a network that pass through the node. If there is more than one shortest path between two nodes, each of which is counted into the total number of shortest paths. To be precise, the definition is as follows:

$$
b(i)=\sum_{j \neq k \neq i} \frac{b_{j k}(i)}{b_{j k}}
$$

where $b_{j k}(i)$ is the number of geodesic paths from $j$ to $k$ containing $i$, while $b_{j k}$ is the total number of geodesic paths linking $j$ and $k$. In Fig. 7, we present the cumulative betweenness distribution 
function $P_{\text {cum }}(b)=\sum_{b^{\prime}=b}^{\infty} P\left(b^{\prime}\right)$ of nodes for the four FTNs. By exploring the cumulative betweenness distributions $P_{\text {cum }}(b)$ of the four FTNs with the method of Clauset et al. [47], like the degree distribution, we find that they follow a power-law function, i.e., $P_{c u m}(b) \sim b^{-(\omega-1)}$, corresponding to the betweenness distribution $P(b) \sim b^{-\omega}$. The exponents $\omega$ for FTN-all, FTN-met, FTN-rub and FTN-oil are 1.871, 1.850, 1.891 and 1.876, respectively. Furthermore, we also make a goodness-of-fit test for each FTN and the results are shown in Table 4. The $p$-value of the goodness-of-fit test for each power-law fit of FTN indicates that the betweenness distribution of each network is consistent with a power-law hypothesis.
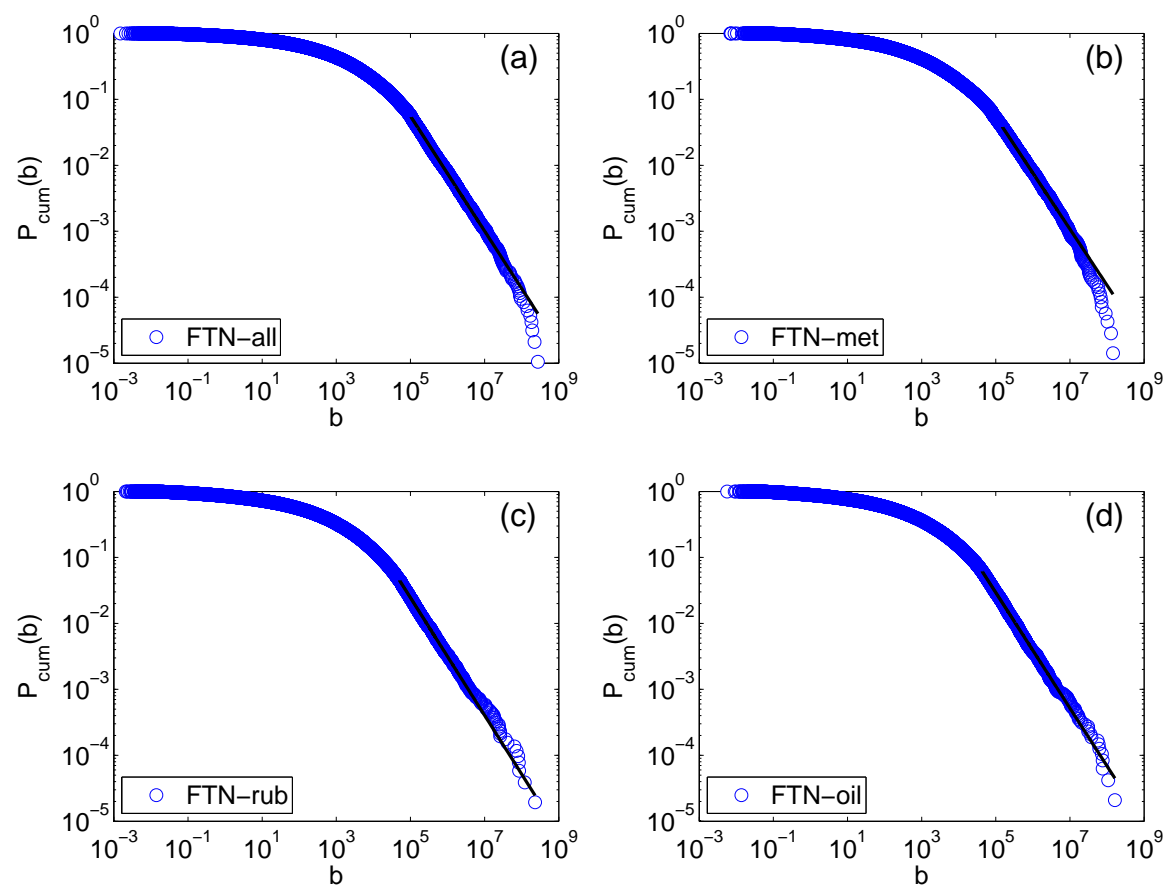

Fig. 7. The cumulative betweenness distributions of the four futures trading networks, which follow a power-law function of $P_{\text {cum }}(b) \sim b^{-(\omega-1)}$. The exponents $\omega$ for FTN-all, FTN-met, FTN-rub and FTN-oil are $1.871,1.850,1.891$ and 1.876 , respectively.

Table 4. The estimations of the lower bound $b_{\min }$ and the exponent $\omega$, and the goodness-of-fit tests of betweenness power-law behaviors of the four futures trading networks. The $p$-values indicate that the betweenness distribution of each network is consistent with a power-law hypothesis.

\begin{tabular}{lccc}
\hline Network & $b_{\min }$ & $\omega$ & $p$-value \\
\hline FTN-all & 103410.8 & 1.871 & 0.237 \\
FTN-met & 149845.9 & 1.850 & 0.437 \\
FTN-rub & 50322.7 & 1.891 & 0.334 \\
FTN-oil & 42603.8 & 1.876 & 0.278 \\
\hline
\end{tabular}


Information communication (e.g. money or asset transfer) exists in FTNs, and money flow is considered as the index of financial markets. The power-law distribution demonstrates that the nodes having high betweenness own such a potential to control information flow passing between node pairs of the network. Accordingly, the participants with high betweenness centrality play decisive role in promoting market boom and enhancing financial function.

\subsection{Disassortative mixing}

In a network, the preference for a node to attach to others that are similar or different in some way is called assortativity, which is often examined in terms of degree correlation between two nodes. Such a correlation is captured by two prominent measures: the assortativity coefficient $[55,56]$ and the average nearest-neighbor degree [57].

The assortativity coefficient is essentially the Pearson correlation coefficient of degree between a pair of linked nodes, and is defined as

$$
r=\frac{1}{\delta_{q}^{2}} \sum_{j k} j k\left(e_{j k}-q_{j} q_{k}\right)
$$

where $q_{k}$ is the distribution of the remaining degree, i.e., the probability that a node has $k$ other edges, $q_{j}$ is the probability that a node has $j$ other edges, $e_{j k}$ is the joint probability distribution of the remaining degrees of the two nodes at either end of a randomly chosen edge, $\delta_{q}$ is the standard deviation of the distribution $q_{k}, \delta_{q}^{2}=\sum_{k} k^{2} q_{k}-\left(\sum_{k} k q_{k}\right)^{2}$. The coefficient $r$ lies in the range $-1 \leq r \leq 1$. Positive $r$ values indicate the existence of assortative mixing pattern, a preference for high-degree nodes to attach to other high-degree nodes, while negative $r$ values imply disassortative mixing, i.e., high-degree nodes to connect, on average, to low-degree ones. Zero $r$ means no assortativity.

For practical evaluation, the assortativity coefficient can also be calculated by:

$$
r=\frac{M^{-1} \sum_{i} j_{i} k_{i}-\left[M^{-1} \sum_{i} \frac{1}{2}\left(j_{i}+k_{i}\right)\right]^{2}}{M^{-1} \sum_{i} \frac{1}{2}\left(j_{i}^{2}+k_{i}^{2}\right)-\left[M^{-1} \sum_{i} \frac{1}{2}\left(j_{i}+k_{i}\right)\right]^{2}}
$$

where $j_{i}$ and $k_{i}$ are the degrees of the nodes at the ends of the $i$ th edge, with $i=1 \ldots M, M$ denotes the number of edges in the network. We calculate the assortativity coefficients of the four FTNs, and obtain the values of $r$, which are $-0.1522,-0.1590,-0.1594$ and -0.1608 for FTN-all, FTNmet, FTN-rub and FTN-oil, respectively. These negative values undoubtedly show that these FTNs are disassortative mixing.

Another means of measuring the degree correlation is to examine the property of neighbor connectivity, i.e., the average nearest-neighbor degree $\left\langle k_{n n}\right\rangle$ of a node with degree $k$, which is defined as

$$
\left\langle k_{n n}\right\rangle=\sum_{k^{\prime}} k^{\prime} P\left(k^{\prime} \mid k\right)
$$

where $P\left(k^{\prime} \mid k\right)$ is the conditional probability that an edge points from a node of degree $k$ to a node of degree $k$ '. If $\left\langle k_{n n}\right\rangle$ increases with $k$, the network is assortative since it implies that the nodes of high degree tend to connect to the nodes of high degree. Conversely, if $\left\langle k_{n n}\right\rangle$ decreases with $k$, the network is disassortative, which means that nodes of high degree are more likely to have nearest neighbors of lower degree. If $\left\langle k_{n n}\right\rangle$ is independent of $k$, there is no correlation between nodes of similar degree. 
In Fig. 8, we plot the neighbor connectivity to depict the overall assortativity trends of the four FTNs. We can see that the FTNs show a power-law dependence on the degree $\left\langle k_{n n}\right\rangle \sim k^{\mu}$, with similar exponent $\mu=-0.52 \pm 0.03$. The result of $\left\langle k_{n n}\right\rangle$ decreasing with $k$ clearly infers the existence of disassortativity in the FTNs.

The disassortative mixing of FTNs discloses that their assortativities are similar to that of technological and biological networks, but are different from social networks. The nontrivial correlation property of the FTNs indicates that the active speculators with high degree do not frequently trade with each other, on the contrary, they tend to make deals with seldom-trading participants. This negative correlation suggests to monitor fluidity risk of the futures market by tracking the active speculators.
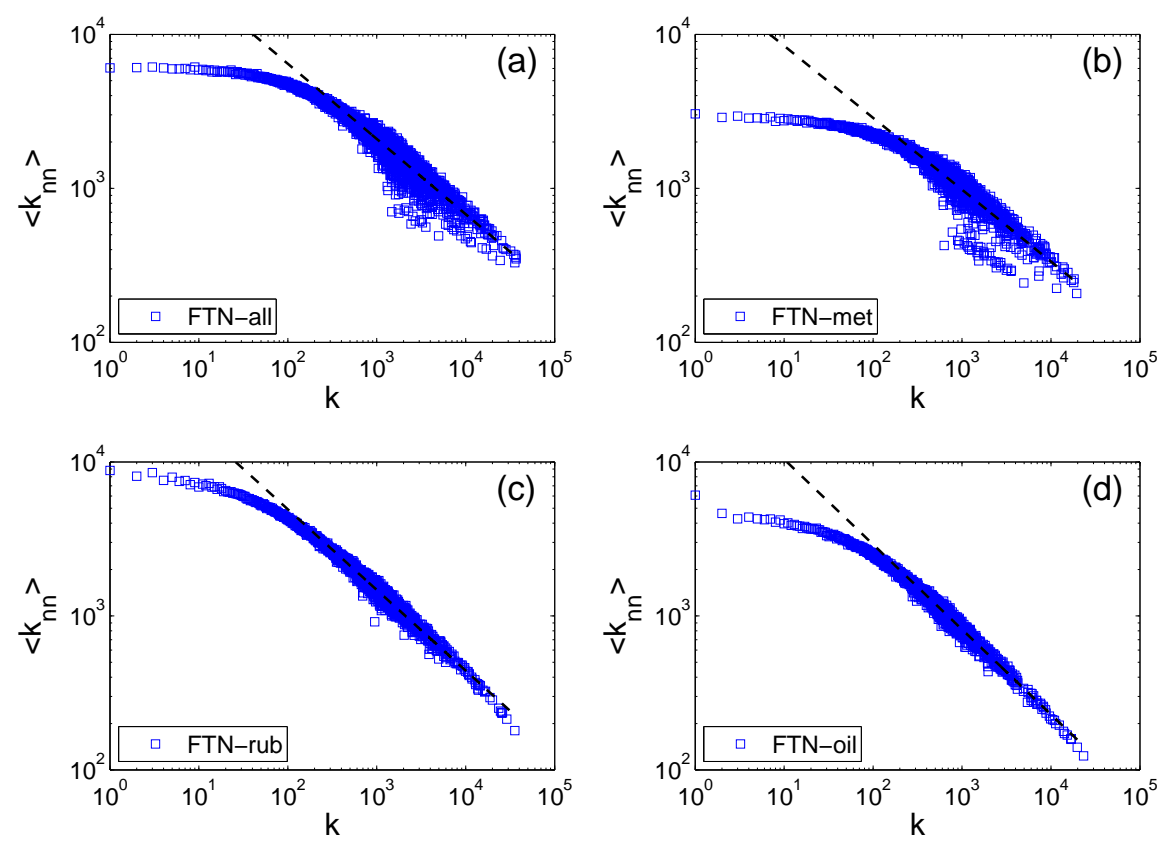

Fig. 8. The average nearest-neighbor degree $\left\langle k_{n n}\right\rangle$ as a function of the node degree $k$ for the four futures trading networks, with the slope of value $-0.52 \pm 0.03$

\subsection{Power-Law Evolution}

Up until now we have explored topological properties of static FTNs. Here we will consider some dynamic characteristics of the FTN-all network by checking its evolutional process, which can characterize the real trading behavior of the futures market.

Like many real-life systems [10, 12, 13], FTN-all also exhibits accelerated growth [58] as shown in Fig. 9(a), which manifests that the number of edges increases faster than the number of nodes. Furthermore, the relationship between the number of edges and the number of nodes in FTN-all follows a power-law function $e(t) \sim n(t)^{\alpha}$. Here, $e(t)$ and $n(t)$ respectively denote the numbers of edges and nodes of the network at time $t$, and $\alpha$ is the exponent. Such a relationship 
is termed the densification power law by Leskovec et al. [59]. We can see that the fitting curve in Fig. 9(a) consists of two segments with different slopes, 1.8 and 3.3, respectively. We notice that the critical point of the broken fitting line is located in the place where the network size is about 30000 , and its corresponding time is exactly the end of the first trading day, which means that the mechanism of intraday growth of edges in FTN-all is totally different from that of the following days.

Besides, we observe that the average degree and density of FTN-all super-linearly grow with the network size, and both have broken lines, as shown in Fig. 9(b) and (c). The changes in both average degree and density over time are directly related to the change of edges' number. The average degree $\bar{k}(t)$ and density $d(t)$ are respectively evaluated by $\bar{k}(t)=2 e(t) / n(t)$ and $d(t)=2 e(t) / n(t)(n(t)-1)$, and thus we obtain $\bar{k}(t) \sim n(t)^{\alpha-1}$ and $d(t) \sim n(t)^{\alpha-2}$. In Fig. 9(b), the two slopes of the broken line are 0.8 and 2.3, respectively, and in Fig. 9(c) they are -0.2 and 1.3, respectively, which explains why the broken line of the density first falls and then rises. According to the empirical viewpoint of Leskovec et al. [59], the changing trend of the average degree of FTN-all in Fig. 9(b) suggests that the network becomes denser as network size increases. However, from the viewpoint of network density based on the benchmark of complete graph, the curve in Fig. 9(c) implies that FTN-all first becomes sparser and then quickly turns denser as network size expands.

Fig. 9(d) shows that the maximum degree $k_{\max }(t)$ of FTN-all grows in power law: $k_{\max }(t) \sim$ $n(t)^{\beta}$ with $\beta=1.7$. This observation indicates that the active participants in the futures market keep vigorous all the time, and the day traders (those who frequently buy and sell futures within the same trading day so that all positions will customarily be closed before the market closes on that day) constitute the core part of the active participants.

In some real complex networks $[1,9]$, the average path length scales logarithmically with the number of nodes, and the diameter slowly grows with the network size. However, in FTN-all we notice that the average path length decreases as the number of nodes increases, so does the diameter. The average path length decays in a power law with a slope of -0.27 in Fig. 9(e), while the diameter of FTN-all decreases as shown in Fig. 9(f). As the network size grows, the simultaneous shrinkage of both the average path length and the diameter in $\mathrm{FTN}$-all provides another evidence of densification in the network.

The exposed properties above indicate that the nodes in FTN-all become closer to each other as the network size grows. These properties indicate the real trade behavior of the futures market. In the futures market, deals come from two sources: the trading by new investors who have just entered the market, and the transactions between the existing speculators. Each new investor makes a few deals for the sake of caution, while the existing speculators, especially the day traders, make a large number of transactions. The latter contribute many deals between the participants who have no trading relationship previously, which results in new links between the nodes that have no direct connections previously in FTN-all, and consequently accounts for the properties mentioned above. Additionally, the different dominant sources of edge addition during network expansion cause the discontiguous slopes of two different fitting lines before and after the switching point, happening at the time when the network size is about 30000 in each logarithmic plot of Fig. 9(a)-(c), and exactly corresponding to the end of the first trading day. In the first trading day, almost all investors are newcomers, so the edge growth originating from new investors is the dominant factor. After that day, the number of new investors and their transactions tend to remain steady, while the edges generated among the existing participants increase rapidly and outnumber those generated by the new investors. The switch between the edge growth's driving factors explains the existence of two different exponents in the power law 
underlying the network's evolution.
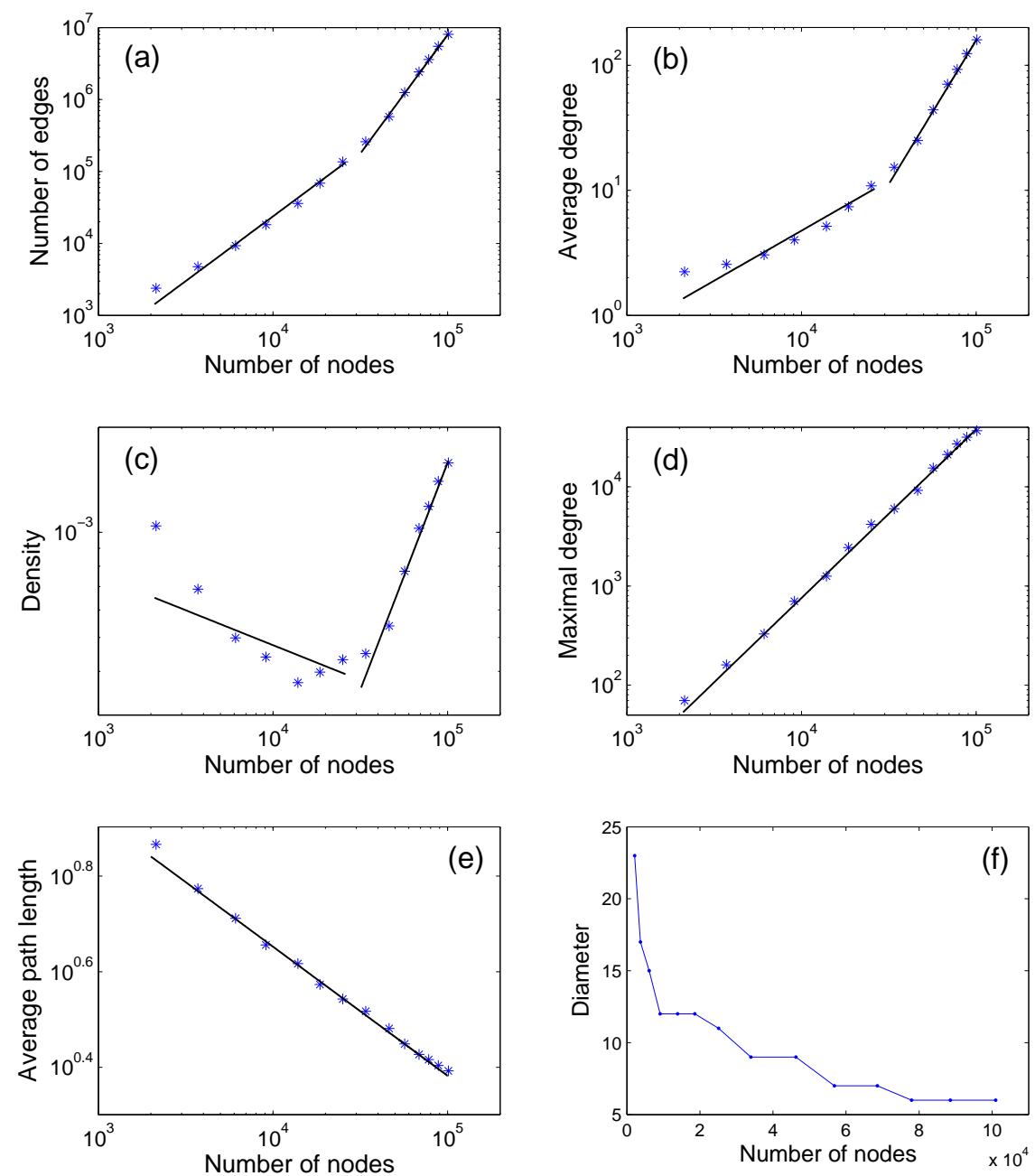

Fig. 9. (a) The number of edges versus the number of nodes in log-log scale for FTN-all; a power law is observed for the slopes of 1.8 and 3.3, respectively in the evolution process. The critical point is at the end of the first trading day. (b) The average degree super-linearly grows in a power law with the slopes of 0.8 and 2.3, respectively. (c) The density changes over time in an approximate power law with slopes of -0.2 and 1.3, which account for the observation that the curve first falls and then rises. The conversion of edge growth's driving factors results in the different slopes in the power law underlying the network's evolution. (d) Maximum degree grows in a power law with regard to the network size, and the slope is 1.7. (e) The average path length decays in a power law with a slope of -0.27 . (f) The diameter decreases as the number of nodes increases. 


\section{Conclusion and Future Work}

In this paper, futures trading networks have been extensively analyzed from the perspective of complex networks. We constructed FTNs by using real trading records covering three months' operation in the Shanghai Futures Exchange. We found a number of interesting statistical properties of the networks, including scale-free behavior, small-world effect, hierarchical organization, power-law betweenness distribution, disassortative mixing and power-law evolution. Some unique features of the FTNs are possibly due to the randomicity of matching counterpart during deal execution and the continuous generation of new links between previously unconnected nodes, which play an important role in determining the structures of futures trade networks. Although three small networks (or sub-networks) are derived from a partition of the whole market dataset, all their statistical properties are consistent with that of the whole market network.

As only undirected graphs are considered in this paper, it is worth further studying the directed FTNs based on trading direction, such as from buyer to seller, which reflects the money flow in the futures markets. In Section 3.1, we simply established the weighted FTNs to demonstrate that active market participants get more active, but it is not enough. Actually, we can construct weighted FTNs or weighted and directed FTNs to investigate the market behaviors of active participants. Moreover, the generation model of FTNs is also an important issue for further exploration, as it can provide valuable insight on financial trade monitoring and risk control.

\section{Acknowledgements}

We thank Prof. Yunfa Hu and Dr. Zhongzhi Zhang for their valuable comments and suggestions. This work was supported by the National Natural Science Foundation of China under Grant numbers 60873040 and 60873070 . Shuigeng Zhou was also supported by the Open Research Program of Shanghai Key Lab of Intelligent Information Processing. Jihong Guan was also supported by the "Shu Guang" Program of Shanghai Municipal Education Commission and Shanghai Education Development Foundation.

\section{References}

\section{References}

[1] D. J. Watts, S. H. Strogatz, Collective dynamics of 'small-world' networks, Nature 393 (1998) 440.

[2] A.-L. Barabási, R. Albert, Emergence of scaling in random networks, Science 286 (1999) 509.

[3] M. E. J. Newman, The structure and function of complex networks, SIAM Rev. 45 (2003) 167.

[4] R. Albert, A.-L. Barabási, Statistical mechanics of complex networks, Rev. Mod. Phys. 74 (2002) 47.

[5] S. Boccaletti, V. Latora, Y. Moreno, M. Chavez, D.-U. Hwang, Complex networks: Structure and dynamics, Phys. Rep. 424 (2006) 175.

[6] L. da. F. Costa, F. A. Rodrigues, G. Travieso, P. R. V. Boas, Characterization of complex networks: A survey of measurements, Adv. Phys. 56 (2007) 167.

[7] C. Song, S. Havlin, H. A. Makse, Origins of fractality in the growth of complex networks, Nature Physics 2 (2006) 275 .

[8] B. A. Huberman, L. A. Adamic, Growth dynamics of the world-wide web, Nature 410 (1999) 131.

[9] R. Albert, H. Jeong, A.-L. Barabási, Diameter of the world-wide web, Nature 401 (1999) 130.

[10] A. Broder, R. Kumar, F. Maghoul, P. Raghavan, S. Rajagopalan, R. Stata, A. Tomkins, J. Wiener, Graph structure in the web, Comput. Netw. 33 (2000) 309.

[11] A.-L. Barabási, R. Albert, H. Jeong, G. Bianconi, Power-law distribution of the world wide web, Science 287 (2000) 2115.

[12] M. Faloutsos, P. Faloutsos, C. Faloutsos, On power-law relationships of the internet topology, Comput. Commun. Rev. 29 (1999) 251. 
[13] H. Jeong, B. Tombor, R. Albert, Z. N. Oltvai, A.-L. Barabási, The large-scale organization of metabolic networks, Nature 407 (2000) 651.

[14] M. E. J. Newman, The structure of scientific collaboration networks, Proc. Natl. Acad. Sci. USA 98 (2001) 404.

[15] A. Barrat, M. Barthélemy, R. Pastor-Satorras, A. Vespignani, The architecture of complex weighted networks, Proc. Natl. Acad. Sci. 101 (2004) 3747

[16] K. Subrahmanyam, S. M. Reich, N. Waechter, G. Espinoza, Online and offline social networks: Use of social networking sites by emerging adults, Journal of Applied Developmental Psychology 29 (2008) 420.

[17] F. Fu, L. H. Liu, L. Wang, Empirical analysis of online social networks in the age of web 2.0, Physica A 387 (2008) 675.

[18] M. Kurant, P. Thiran, Layered complex networks, Phys. Rev. Lett. 96 (2006) 138701.

[19] B. B. Su, H. Chang, Y.-Z. Chen, D. R. He, A game theory model of urban public traffic networks, Physica A 379 (2007) 291.

[20] R. Guimerà, L. A. N. Amaral, Modeling the world-wide airport network, Eur. Phys. Jour. B 38 (2004) 381.

[21] W. Li, X. Cai, Statistical analysis of airport network of china, Phys. Rev. E 69 (2004) 046106.

[22] H.-K. Liu, T. Zhou, Empirical study of chinese city airline network, Acta Physica Sinica 56 (2007) 106

[23] D.-D. Han, J.-H. Qian, J.-G. Liu, Network topology and correlation features affiliated with european airline companies, Physica A 388 (2009) 71.

[24] A. E. Motter, A. P. S. de Moura, Y.-C. Lai, P. Dasgupta, Topology of the conceptual network of language, Phys. Rev. E 65 (2002) 065102

[25] S. M. G. Calderia, T. C. P. Lobão, R. F. S. Andrade, A. Neme, J. G. V. Miranda, The network of concepts in written texts, Eur. Phys. J. B 49 (2006) 523

[26] S. G. Zhou, G. B. Hu, Z. Z. Zhang, J. H. Guan, An empirical study of chinese language networks, Physica A 387 (2008) 3039.

[27] R. Albert, A.-L. Barabási, Topology of complex networks: Local events and universality, Phys. Rev. Lett. 85 (2000) 5234.

[28] G. Bianconi, A.-L. Barabási, Competition and multiscaling in evolving networks, Europhys. Lett. 54 (2001) 436.

[29] C. P. Zhu, S. J. Xiong, Y. J. Tian, N. Li, K. S. Jiang, Scaling of directed dynamical small-world networks with random responses, Phys. Rev. Lett. 92 (2004) 218702.

[30] L. K. Gallos, R. Cohen, P. Argyrakis, A. Bunde, S. Havlin, Stability and topology of scale-free networks under attack and defense strategies, Phys. Rev. Lett. 94 (2005) 188701.

[31] M. E. J. Newman, A measure of betweenness centrality based on random walks, Social Networks 27 (2005) 39.

[32] M. Tumminello, T. Aste, T. Di Matteo, R. N. Mantegna, A tool for filtering information in complex systems, Proc. Natl. Acad. Sci. USA 102 (2005) 10421.

[33] J.-G. Liu, Y.-Z. Dang, Z.-T. Wang, Complex network properties of chinese natural science basic research, Physica A 366 (2006) 578

[34] J.-G. Liu, Z.-G. Xuan, Y.-Z. Dang, Q. Guo, Z.-T. Wang, Weighted network properties of chinese nature science basic research, Physica A 377 (2007) 302.

[35] L. da F. Costa, O. N. Oliveira Jr., G. Travieso, F. A. Rodrigues, P. R. V. Boas, L. Antiqueira, M. P. Viana, L. E. C. da Rocha, Analyzing and modeling real-world phenomena with complex networks: A survey of applications.arXiv:0711.3199.

[36] R. N. Mantegna, Hierarchical structure in financial markets, Eur. Phys. J. B 11 (1999) 193.

[37] G. Bonanno, G. Caldarelli, F. Lillo, R. N. Mantegna, Topology of correlation-based minimal spanning trees in real and model markets, Phys. Rev. E 68 (2003) 046130.

[38] J.-P. Onnela, A. Chakraborti, K. Kaski, J. Kertész, A. Kanto, Asset trees and asset graphs in financial markets, Phys. Scr. T106 (2003) 48.

[39] J.-P. Onnela, K. Kaski, J. Kertész, Clustering and information in correlation based financial networks, Eur. Phys. J. B 38 (2004) 353.

[40] R. K. Pan, S. Sinha, Collective behavior of stock price movements in an emerging market, Phys. Rev. E 76 (2007) 46116.

[41] D. Garlaschelli, S. Battiston, M. Castri, V. D. P. Servedio, G. Caldarelli, The scale-free topology of market investments, Physica A 350 (2005) 491

[42] S. Battiston, J. F. Rodrigues, H. Zeytinoglu, The network of inter-regional direct investment stocks across europe, Adv. Complex Syst. 10 (2007) 29.

[43] M. Franke, B. Hoser, J. Schröder, On the analysis of irregular stock market trading behavior, GfK1 (2007) 355.

[44] S. C. Wang, J. J. Tseng, C. C. Tai, K. H. Lai, W. S. Wu, S. H. Chen, S. P. Li, Network topology of an experimental futures exchange, Eur. Phys. J. B 62 (2008) 105.

[45] Z.-Q. Jiang, W.-X. Zhou, Complex stock trading network among investors, Physica A 389 (2010) 4929.

[46] M. E. J. Newman, Power laws, pareto distributions and zipfs law, Contemp. Phys. 46 (2005) 323.

[47] A. Clauset, C. R. Shalizi, M. E. J. Newman, Power-law distributions in empirical data, SIAM Review 51 (2009) 
661.

[48] A. Barrat, M. Barthélemy, A. Vespignani, Modeling the evolution of weighted networks, Phys. Rev. E 70 (2004) 066149.

[49] K.-I. Goh, B. Kahng, D. Kim, Nonlocal evolution of weighted scale-free networks, Phys. Rev. E 72 (2005) 017103.

[50] W.-X. Wang, B.-H. Wang, B. Hu, G. Yan, Q. Ou, General dynamics of topology and traffic on weighted technological networks, Phys. Rev. Lett. 94 (2005) 188702.

[51] Q. Ou, Y.-D. Jin, T. Zhou, B.-H. Wang, B.-Q. Yin, Power-law strength-degree correlation from resource-allocation dynamics on weighted networks, Phys. Rev. E 75 (2007) 021102.

[52] Z. Zhang, S. Zhou, L. Fang, J. Guan, Y. Zhang, Maximal planar scale-free sierpinski networks with small-world effect and power-law strength-degree correlation, Europhysics Letters 79 (2007) 38007.

[53] E. Ravasz, A.-L. Barabási, Hierarchical organization in complex networks, Phys. Rev. E 67 (2003) 026112.

[54] L. C. Freeman, A set of measures of centrality based on betweenness, Sociometry 40 (1977) 35.

[55] M. E. J. Newman, Assortative mixing in networks, Phys. Rev. Lett. 89 (2002) 208701.

[56] M. E. J. Newman, Mixing patterns in networks, Phys. Rev. E 67 (2003) 026126.

[57] R. Pastor-Satorras, A. Vázquez, A. Vespignani, Dynamical and correlation properties of the internet, Phys. Rev Lett. 87 (2001) 258701.

[58] S. N. Dorogovtsev, J. F. F. Mendes, Effect of the accelerating growth of communications networks on their structure, Phys. Rev. E 63 (2001) 025101.

[59] J. Leskovec, J. Kleinberg, C. Faloutsos, Graph evolution: Densification and shrinking diameters, ACM Trans. Knowl. Discov. Data 1 (2007) 2. 